\title{
A Survey on Internet of Things (IoT), Industrial loT (IloT) and Industry 4.0
}

\author{
Sujit N. Deshpande \\ Research Scholar, \\ Department of Computer Science and Engineering, \\ KLS, Gogte Institute of Technology, Belagavi \\ Assistant Professor, \\ Department of Computer Engineering, \\ PES's, Modern College of Engineering, Pune
}

\author{
Rashmi M. Jogdand, PhD \\ Research Supervisor and Professor \\ Department of Computer Science and Engineering \\ KLS, Gogte Institute of Technology, Belagavi
}

\begin{abstract}
Internet of Things (IoT) is one of the emerging technologies comprises of 'things' capable of identifying, sensing, communicating, processing, and actuating the data gathered from the environment. IoT plays an important role in various sectors including healthcare, medical, education, smart cities, and also it contributes in developing smart industries as part of the industrial revolution 'Industry 4.0' with the use of Industrial Internet of Things (IIoT) technology. This paper provides an in-detail overview of IoT with its functioning, architecture, applications, middleware framework, protocols, and its integration with other key enabling technologies. This paper also provides an overview of the industrial revolution industry 1.0 to 4.0 and the Industrial Internet of Things (IIoT) with its applications, architecture, and messaging protocols. Finally, paper covers research challenges in IoT and IIoT.
\end{abstract}

\section{Keywords}

IoT, IIoT, Industry 4.0.

\section{INTRODUCTION}

Technology, day by day is moving at a fast pace. Disruptive technologies are moving even faster. Things (objects), to make them live, smart and intelligent, are connected to the internet; they realize the idea of the Internet of Things (IoT) a term initially coined by Kevin Ashton in the year 1999. Since then many researchers and educationists have been continuously proving their efforts towards developing smart IoT applications in different sectors including healthcare, medical, education, smart cities, industries, transportation, and many more. This introduces many research challenges and issues in accomplishing the design and development of the planned applications. There is a need for addressing various research challenges and issues in IoT. Researchers have addressed various challenges and issues in various areas of IoT include IoT architecture, communication protocols, identification, sensing, storage, processing, security, and services.

In the coming years, the focus will be on millions of devices interacting with one another with minimal human intervention, to make business and processes more efficient, effective, and transparent. The Internet of Things (IoT) plays a remarkable role and improves the quality of our lives. It has many definitions acceptable by world community users. It can be defined as "An open and comprehensive network of intelligent objects that can auto-organize, share information, data, and resources, reacting and acting in face of situations and changes in the environment"[1].
From home automation, transportation, and wearable devices to industry automation IoT is expected to transform how we work, live, and do our day to day activities. IoT is now not only limited to applications that make a change to only an individual but has now grown up to make industries smarter. This talks about the smart use of IoT in industries, which is named Industrial IoT (IIoT) where IoT is used to develop industrial applications using various sensors and wireless devices. To cater to the high demands and infrastructural needs of industries, IoT is widely used in industry to make an industry a smart one, can be named as a smart factory [2]. This fulfills the demand of the 4th industrial revolution termed as 'Industry 4.0' [3 - 4]. IoT and Cyber-Physical Systems (CPS) are key elements of Industry 4.0. Along with IIoT, artificial intelligence, big data, cloud computing, cyber security, system integration, simulation, augmented reality, and additive manufacturing are pillars of industry 4.0 [3 - 4].

In this paper, we have tried to address the details of IoT, IIoT, and Industry 4.0. The paper is organized as follows: In section II we have provided market overview and opportunities in IoT. Section III overall covers the details of the IoT includes IoT functioning, IoT Architecture, IoT Applications, IoT middleware framework, IoT standards, and protocols and concluded with IoT in support with many key enabling technologies. It helps researchers to understand and know about IoT. Section - IV presents Industry 4.0 with the industrial revolution, future trends in industrial communication, key technologies of Industry 4.0, and research challenges in it. IIoT presented in Section - V covers the introduction of IIoT, its architecture, ecosystem, and key enabling technologies. Research challenges in IoT and IIoT are discussed in Section - VI. Finally, Section - VII concludes the paper.

\section{MARKET OVERVIEW AND OPPORTUNITY}

In the technological environment, IoT and IIoT have changed the approach towards the use of devices and things. IoT not only connects things (objects) but also includes services and solutions. The developing pattern of internet-enabled gadgets and rising need for internet is a portion of the main considerations which are foreseen the positive development of IoT. Advancements in semiconductor technologies play a vital role in the development of lightweight, efficient and smarter devices than the normal and heavy devices. Rapid advancements in manufacturing, electronics, and IT sectors across the world are gaining attraction from consumers which successively increases the demand for the internet of things (IoT). Increasing usage of the internet in conjunction with the 
rising penetration of smartphone users around the world is one of the prime factors behind the positive growth of the industry. "The IoT market reached USD 598.2 billion in 2015 and the market is expected to reach USD 724.2 billion by 2023"[5].

Geographically, Asia-Pacific is predicted to showcase incredible growth within the market of the internet of things (IoT) across the world shortly. The market of the Asia-Pacific region acquired $36 \%$ of the global revenue share in 2015 and the market is anticipated to grow at a CAGR of $10.2 \%$ during the forecast period i.e. 2016-2023 [5]. The internet of things (IoT) comprises connected vehicles, wearable electronic devices, and other electronic appliances that will be connected through the internet and operate from remote locations. Widescale availability and variety in technological devices are providing ease to the consumers to adopt the latest technology. Increasing technically competent populations across the globe is increasing the demand for the internet of things (IoT) globally.

The market potential of the Internet of Things in India alone is expected to reach USD 9 billion by 2020 [5]. With this, the IoT units in India can also see the rapid growth of 31 times to reach 1.9 billion by 2020 [5]. At present, there are approximately 7.6 billion people in the world, nearly 3.7 billion of those are connected to the Internet; roughly $50 \%$ of such connected population resides in Asia and $24 \%$ amongst them belongs to India [6].

Internet of things (IoT), with the potential to extend the connectivity of everything, everyone and everywhere, is changing the way we do things. India with a landscape of 120 IoT firms has the specified technical skills to drive the IoT revolution making it one among the key countries poised for the implementation of large scale IoT projects [7]. As per a joint report by IAMAI (The Internet and Mobile Association of India) and Deloitte, Industrial IoT is predicted to surpass the buyer IoT space in India by 2020 . It also predicts a $\$ 12$ billion IoT opportunity. The accelerated pace of IoT adoption within the country will make sectors like industrial manufacturing, energy, agriculture, utilities, transport, and logistics lead the market [8].

\section{INTERNET OF THINGS}

\subsection{IoT Functioning}

This section explains the functioning of the IoT. The Internet has allowed its' users to change their way of work and communicate with each other by providing sound connectivity. Using this connectivity, IoT is trying to connect multiple devices at a time to the internet thereby making them smart and intelligent to facilitate man to machine and machine to machine interactions. This basic approach of functioning IoT has covered many areas, includes home automation, vehicle automation, industrial automation, healthcare and medical, retail, education, and many more. Below is the IoT ecosystem, which explains the working of IoT.

\subsubsection{Identifying and Sensing}

Identification of objects (things) with its id and address is crucial for IoT. There are identification methods such as electronic product codes (EPC) and ubiquitous codes (uCode) [10]. Sensors collect and transmit the data to the cloud or data warehouse. Context-awareness is possible using sensor technology. Further, it is analyzed to take the required actions based on demand. Sensors can be classified as passive and active sensors, analog and digital sensors, and scalar and vector sensors. Some of the examples of these sensors are an accelerometer, water level, temperature sensor, light detection sensor, analog pressure sensor, magnetic sensor, passive infrared sensor, digital temperature sensor, magnetic field, motion detector sensor, and smartphone - a generic sensor [17] with many types of sensors embedded in it such as the location sensor (GPS), movement sensors (accelerometer, gyroscope), camera, light sensor, microphone, proximity sensor, and magnetometer.

\subsubsection{Communication}

Data collected through several sensors is sent to the cloud for further processing, but it requires a way to reach there. Devices (things) with sensors can be connected to the cloud using various technologies available as, cellular, WiFi, satellite, Bluetooth, low power wide area network (LPWAN). The connection is established by using a gateway or router or directly to the internet via Ethernet. Based on the IoT application scenario the connectivity using the abovementioned technologies is decided that suits better though every technology has tradeoffs concerning power consumption, bandwidth, and range.

\subsubsection{Computation and Data Processing}

Collected data through various sensors is processed to extract information from the data. Usually, this is carried out at the cloud with the help of intelligent software. Comparing data across broad areas becomes efficient with the help of the cloud. To perform several computations processing elements like microcontrollers, microprocessors, and software applications are essential in IoT. Various hardware platforms like Particle.io, Espressif ESP8266 Board, Intel IoT Development Board, Adafruit.io, Arduino, Raspberry PI, BeagleBone are available for computations. Different software platforms like 2lemetry - IoT Analytics Platform, Appcelerator, AWS IoT platform, Bosch IoT Suite - MDM IoT Platform, Ericsson Device Connection Platform (DCP) MDM IoT Platform, EVRYTHNG - IoT Smart Products Platform, IBM IoT Foundation Device Cloud, ParStream IoT Analytics Platform, PLAT.ONE - end-to-end IoT and M2M application platform, ThingWorx - MDM IoT Platform, Xively- PaaS enterprise IoT platform [11] are available. TinyOS, Contiki, LiteOS, Riot OS, and Android are some of the operating systems used in IoT systems [10].

\subsubsection{User Interface}

It is an important element of the IoT ecosystem. It makes information available to the end-user. This could be done through an alert in the form of an email or notification. Users may use a web application or mobile application to respond to the system as per need.

\subsection{IoT Architecture}

IoT makes it possible to have an interaction between the physical and digital world using sensors and actuators. The objective of IoT is to help everyone ease their task and day to day challenges. IoT aims to create a world of billions or trillions of heterogeneous objects, devices, and appliances that are interconnected through a network and used collaboratively to achieve complex tasks that require a high degree of intelligence. But when IoT can be thought of as a solution to handle a problem in any sector, it has to cater to different requirements and needs. It introduces a need for having flexible layered architectures. The multilayer architectures are presented in [6], [7], [8]. Below are some of the multilayer architectures explained with layers and functioning of each one. 


\subsubsection{Three Layer Architecture}

Typically, the IoT architecture has 3 layers; perception layer, network layer, and application layer, which are described below -

Perception layer - Sensors and actuators are the main entities of this layer. Sensing and gathering the information from the environment and also identifying the other objects in the environment are the tasks performed by the various types of sensors available in a variety of applications [9]. Commonly available sensors in the market are a temperature sensor, proximity sensor, pressure sensor, and optical sensors.

Network layer- This layer is responsible for connecting smart things, network devices, and servers. Also used for transmitting and processing sensor data.

Application layer - This layer is responsible for delivering application-specific services to the user. It also defines applications in which IoT can be deployed.

\subsubsection{Five Layer Architecture}

With this basic 3 layer architecture, there are various IoT architectures such as middleware based, SOA based, and 5 layer architecture introduced in [10]. For smooth functioning of the system and each component to carry out the smoother operation, the 3 layer architecture is expanded to 5 layer architecture, consisting of the Perception layer, transport layer, processing layer, application layer, and business layer. The functioning of the perception layer is same as that of in 3 layer architecture. The functioning of the remaining layers is discussed below -

Transport layer - It transfers the gathered data by the sensors between different layers through a network for further processing through any medium like $3 \mathrm{G}$, wireless network, Bluetooth, RFID, or NFC.

Processing layer - It mainly does the task of storing and processing the data. It performs analysis as well as processes a huge amount of data as per users' needs. Databases, cloud computing services, big data processing, and data analytics modules come into the picture as a part of the processing layer.

Application layer - Followed the processing of data, produced information is given to the application layer, responsible for delivering application-specific services to the user.

Business layer - It is the topmost layer in 5 layer IoT architecture. It manages the whole IoT system including applications, business and profit models, and users' privacy [10]. It is responsible to build a specific business model as per the need and requirements. Most of IoT applications are built considering this 5 layer architecture [10].

\subsection{IoT Applications}

IoT plays a vital role in many real-life applications. Some of the applications of IoT include [10], smart homes [12], [13], wearable devices [14], industrial automation [15], [16], smart transportation systems, health care, smart retail, smart farming - agriculture. IoT can effectively be used in smart city services includes health care, transportation, and governments. IoT can be effectively used in prevention of geologic hazards [50]. IoT has a major role in 5G systems and it introduces 5G IoT [47].

\subsection{IoT middleware framework}

The IoT middleware framework connects different components; that help to have smooth communication between various devices. The interface facilitates the interaction between the "Internet" and the "Things", which means hardware or applications. IoT framework provides cloud support and all other needs the IoT technology should satisfy. IoT system will need cloud services, security for data stored in the cloud, supporting protocols, edge devices such as gateways, and supporting software. An IoT framework can be used to simplify programming work.

\subsection{IoT Standards and Protocols}

Connected devices in the IoT ecosystem are expanding over the last two decades. These devices are enabled to collect, process, and send data to other objects, applications, or servers. It covers many areas of applications including manufacturing, medicine, automotive, security systems, transportation, and many more. The IoT devices are safely connected to a common network, allows the IoT system to function and transfer data. IoT standards and protocols make such a connection possible.

IoT devices are powered by batteries; devices are also having low computing capability and storage capacity. To overcome these constraints IoT applications have to rely more on a cloud for computing and storage purposes. So communication challenges involved in it are identification with unique addressing, routing protocols, and high-speed reliable communication with low power.

To address above mentioned challenges various communication technologies have been proposed includes Bluetooth, RFID and Near Field Communication (NFC) [18] and protocols have also been developed for different layers are listed below - Physical and MAC layer - IEEE 802.15.4 [21] and Zigbee [21], adaptation layer - 6LoWPAN [19], network layer - A routing protocol (RPL) [20], transport layer - UDP a connectionless protocol and application layer [10] CoAP [22 - 23] (Constrained Application Protocol), MQTT [24] (Message Queue Telemetry Transport), XMPP [25] (Extensible Messaging and Presence Protocol), AMQP [26] (Advanced Message Queuing Protocol) and DDS [27] (Data Distribution Service).

\subsection{IoT - integration with key enabling technologies}

IoT undoubtedly is one of the most exciting topics to the research community, the public sector, and industry facilitates communication between all sorts of connected "things" into a comprehensive network of interrelated computing intelligence without the intervention of a human. IoT can be used to solve many real-time applications in various areas. But it also brings several implementation challenges. These can be overcome with the integration of several key enabling technologies discussed below. Many applications can be found with the integration of IoT with one or more technologies listed below. Below are key enabling technologies discussed that can be integrated with IoT to implement various real-time applications.

\subsubsection{Cloud Computing}

Cloud can be integrated with IoT though these are two different technologies [48]. Integration of Cloud and IoT, given the name CloudIoT [28] has given birth to many applications includes healthcare, education, smart cities, and medical [29]. On-demand of users cloud computing provides a service. It can be provided in terms of platform, infrastructure, and software as a service. Sensors have limited memory and processing power it can only store and process local data. Cloud can be effectively used by IoT applications to monitor and analyze objects. It also supports artificial 
intelligence for the decision making, thus bypassing human intervention.

\subsubsection{Big Data Analytics}

Since the number of devices (objects) connected to the internet is increasing day by day, data generated by these devices also growing at a faster rate. To fulfill the need IoT and Big Data should have to go hand in hand. Big Data manages the huge amount of data generated using its technologies. The Internet of Things (IoT) and big data are two vital subjects in commercial, industrial, and many other applications. The name IoT was framed in approximately a decade ago and refers to the world of machines or devices connected to the Internet, by which a large amount of big data is collected, stored, and managed. Big data additionally refers to the analysis of this generated data to produce useful results. The main motivating power behind the IoT and big data have been the collection and analysis of data related to consumer activities to find out why and what customers buy.

\subsubsection{AI and $M L$}

The present business world is changing with the use of IoT (Internet of Things). IoT is helping in collecting a huge amount of data from multiple sources. This huge amount of data coming from several IoT devices makes it difficult to collect, process, and analyze the data. Combining AI (Artificial Intelligence) and ML (Machine Learning) with IoT can solve the issue. The integration of AI and ML with IoT can create intelligent machines that simulate smart behavior and supports in decision making with little or no human interaction.

\subsubsection{Image Processing}

There are various applications with integration of IoT in image processing exists includes image processing in medical applications, image processing in IoT based smart agriculture, smart city system, smart traffic control, etc. [43]. Smart agriculture application with integration of IoT and image processing is presented in [44].

\subsubsection{Augmented Reality}

The gap between the physical and digital world, one of the great promises of industry 4.0, can be easily bridged by integrating IoT with Augmented Reality (AR). It guarantees business benefits such as augmentation and standardization and customization and efficiency [41]. The integration of IoT with AR helps in e-learning and develops an interest in learning among students [42].

\section{INDUSTRY REVOLUTION - INDUSTRY 1.0 TO 4.0}

The industrial revolution, since the first revolution, resulted in radical changes in manufacturing, from water and steampowered machines to electrical and digital automated production. Complicated, automatic, and sustainable manufacturing processes make it easy for people to operate machines simply, efficiently, and persistently [30].

The four industrial revolutions are shown in figure 1.

\subsection{Industry 1.0}

Industry $1.0 \mathrm{can}$ also be deemed as the beginning of the industry culture which focused equally on scale and efficiency. The first weaving loom was introduced in 1784, with an increase of productivity in efficiency and scale. Water and steam were used in the first industrial revolution around 1760 with the transition to new manufacturing processes. It has benefited in manufacturing a larger number of various goods and creating a better standard of living for some. The textile industry, especially, was transformed by industrialization, as was transportation. Fuel sources like steam and coal made machine use more feasible, and therefore the idea of producing with machines quickly spread. Machines allowed faster and easier production, and that they made all types of latest innovations and technologies possible also [31].

\subsection{Industry 2.0}

The beginning of the 20th century marked the start of the second industrial revolution. In this revolution, machines were running on electrical energy. The main contributor to this revolution was the development of machines running on electrical energy. It is more efficient to work and maintain both in terms of cost and energy, unlike water and steam based machines which were comparatively inefficient and resource hungry. This era also saw the evolution of the industry culture introduced in industry 1.0 into the management program to reinforce the efficiency of producing facilities. American mechanical engineer Fredrick Taylor introduced the study of approaches to optimize worker, workplace technique, and optical allocation of recourses [32]. The second industrial revolution picked up in the period between the 1760 s to 1840 s as "Technological revolution" referred by historians and mainly was in Britain, Germany, and America [31].

\subsection{Industry 3.0}

This technological revolution was spurred by the advances within the electronic industry within the previous couple of decades of the 20th century. Industry 3.0 may be a huge leap ahead wherein the arrival of computer and automation ruled the economic scene. There have been more robots to perform human tasks like programmable logic controllers, washing machines, etc. To automate production around 1970 this revolution started using electronics and IT (Information Technology). This has introduced the use of Programmable Logic Controllers (PLC) to automate the human tasks on the assembly line [31].

\subsection{Industry 4.0}

The boom in the internet and telecommunication industry in the 1990s revolutionized in the way we connected and exchanged information. It's resulted in paradigm changes in the manufacturing industry and traditional production operation merging the boundaries of the physical and the virtual world. Industry 4.0 using a cyber-physical system to analyze, share and guide intelligent action for various processes in the industry to make the machine smarter.

In Industrial Internet of things (IIoT) the exchange of information is possible without human intervention by smart machines, storage systems, and production facilities [31]. The Cyber-Physical Systems (CPS), IoT, cloud computing, and cognitive computing are key elements of industry 4.0 [31].

Industry 4.0 fosters exactly what may be known as the "smart factory". Inside modular organized wise industrial facilities, cyber-physical systems keep track of bodily procedures, produce a digital duplicate from the bodily globe as well as help to make decentralized choices. Within the internet of things, cyber-physical systems connect as well as work with one another with people within real-time each in the house as well as throughout organizational services provided as well as utilized by individuals from the worth string.

The reference model of industry 4.0 as RAMI 4.0 is discussed in [33]. The various key technologies of Industry 4.0 include 
the IIoT, Cloud Computing, Big Data, Simulation,

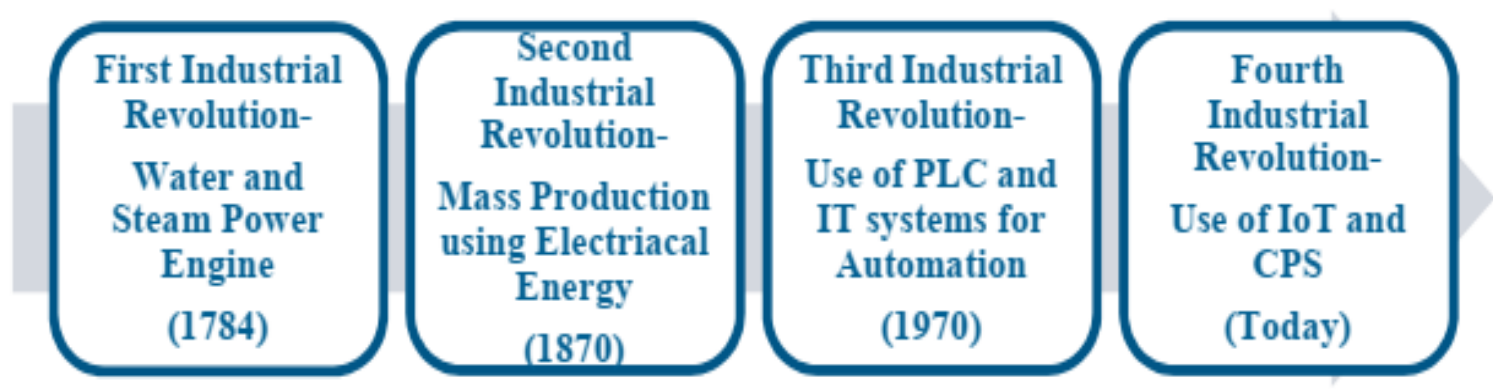

Fig 1: Four Industrial Revolutions [34].

Augmented Reality, Additive Manufacturing, Horizontal and Vertical Systems Integration, Autonomous Robots, and Cyber Security are discussed in [33]. The various issues and challenges of industry 4.0 are discussed in [34]. This industrial revolution has started in Germany to boost its economy.

\section{INDUSTRIAL INTERNET OF THINGS (IIOT)}

The transformation of industrial processes using various technologies such as Cyber-Physical Systems (CPS), Internet of Things (IoT), Cloud Computing, Machine Learning, and Data Analytics is the main concern in Industry 4.0. IIoT is becoming popular in the industrial sector to improve the personal condition in the industry, to improve operational efficiency, and to increase the machine lifetime.

\subsection{IoT Applications in Industry}

IoT plays a vital role in industrial applications. Its use is growing in various applications including production and manufacturing, health care services, transportation, and many more [35]. The use of IoT in the healthcare service industry, food supply chain, safer mining production, transportation and logistics, firefighting, etc are discussed in detail in [35]. In manufacturing and production industries the IoT can be effectively used in predictive maintenance [49].

\subsection{Messaging protocols for IIoT}

To support heterogeneity and to ensure application interoperability IoT uses messaging protocols with the presence of broker. More commonly known messaging protocols are Message Queuing Telemetry Transport (MQTT) and Advanced Message Queuing Protocol (AMQP) [36]. A comparative study between MQTT and MODBUS TCP is presented in [37]. Overview of IIoT technology, key enabling technologies, and their applications in industries are discussed by [38].

\subsection{IIoT Architecture}

IIoT three-tier system architecture with its layers as physical, communication, and application, is described in detail by [39]. It can also be represented in three layers with its layers as enterprise - business applications, platform - for secure communication, and edge - monitoring and control [51].

Cloud computing, hybrid cloud, and edge computing can be the various computing systems in IIoT [39]. Different open research challenges and necessary future research directions in IIoT are well presented in [39]. Security is the measure of concern in IIoT. Supervisory Control and Data Acquisition (SCADA) systems are used to provide security in IIoT [40].

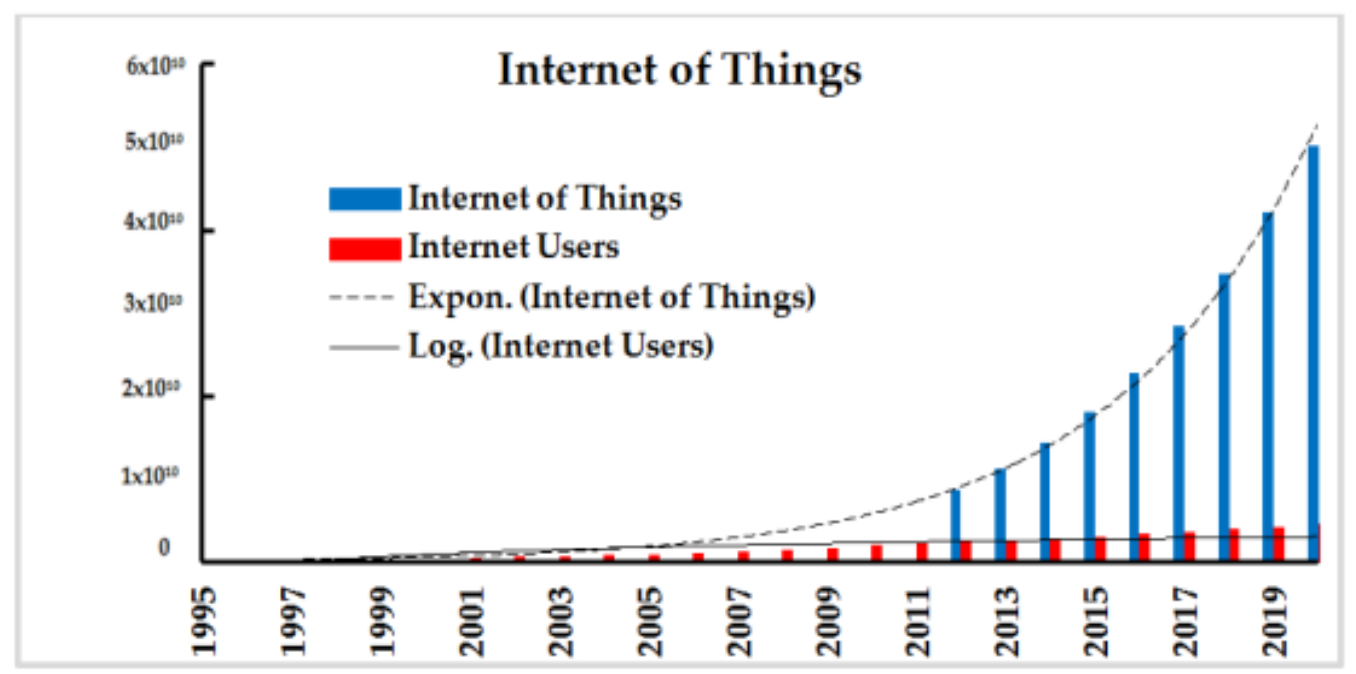

Fig 2: Growth of IoT [46] 


\subsection{Examples of IIoT along with their applications}

IIoT fulfills the requirements of industry 4.0 , which is a type of industry framework used by many industries for their applications including MAN, Siemens, Caterpillar (CAT), Airbus, ABB, Fanuc, Magna Steyr, John Deere, Tesla, Hortilux [45].

\section{RESEARCH CHALLENGES IN IOT AND IIOT}

Since IoT and IIoT are still in the developing stage, there is still huge scope for these technologies to be mature enough such that to be fully functional and trusted technologies. Many issues that need to be addressed include architecture, lack of standardization, issues of interoperability, security, scalability, etc. There are many solutions proposed by researchers but these solutions are not generic, they either address challenges only in a specific domain or targeted towards distinct objectives. As the research in IoT is advancing, new application domains are being discovered with increased complexity in implementation.

There is continuous growth in IoT. Figure 2 shows the growth of IoT concerning internet users and connected devices. So by considering this it is clear that there is still a need to address the research challenges in the different areas of IoT includes,

\subsection{Monitoring and Sensing}

In IoT huge amount of data is gathered through various sensors. Several key challenges related to gathering of the data include battery lifetime of the devices and power consumption issues as continuous sensing and transmission of data is essential as a part of any application. Though technologies have progressed at a certain level they continue to evolve for more energy efficiency.

\subsection{Communication Protocols}

There are several protocols available in IoT, includes CoAP, MQTT, etc. These protocols are available as a single protocol. Though researchers contributed to the development of these protocols there is still scope in the direction of generalized support for various protocols.

\subsection{Data management and processing}

The data management, processing, and analysis are complex in IoT because of large scale data and heterogeneity. Various cloud-based systems exist, focuses more on centralized processing. To overcome the limitations of existing cloud architectures of not meeting data transmission rates for IoT data, fog, and mobile cloud computing technologies can be used with research challenges that need to be addressed.

\subsection{Security and privacy}

This is one of the important challenges in IoT that needs major attention from researchers to provide strong security and data privacy to avoid attacks on IoT applications. The main aim is to ensure the security and confidentiality of the users, infrastructures, data, and devices of the IoT, and guarantee the availability of the services. Thus, research in IoT security is gaining much more attention from researchers.

IIoT is adopted by most of the manufacturers, companies, healthcare providers to improve productivity and efficiency through various features supported by IIoT. IIoT has many research challenges that need to be addressed in future research. Some of the challenges of IIoT are real-time performance, security, and privacy, energy efficiency, coexistence, and interoperability.

\section{CONCLUSION}

In this paper, we reviewed, IoT, as one of the emerging technologies, IIoT, and Industry 4.0 in detail including architectures, functioning, protocols, and various technologies integration with IoT to build smart applications. This paper covers market overview and opportunities in IoT. The industrial revolution starting from industry 1.0 to 4.0 is covered in this paper. This paper also covers IIoT with its architecture, applications, messaging protocols, and examples. Finally, we discussed the research directions to address challenges.

\section{REFERENCES}

[1] Madakam, Somayya\&Ramaswamy, R \&Tripathi, Siddharth. (2015). Internet of Things (IoT): A Literature Review. Journal of Computer and Communications.3. 164-173. 10.4236/jcc.2015.35021.

[2] $\mathrm{Xu}, \mathrm{Li} \& \mathrm{He}, \mathrm{Wu} \& \mathrm{Li}$, Shancang. (2014). Internet of Things in Industries: A Survey. IEEE Transactions on Industrial Informatics. $\quad 10 . \quad 2233-2243$. 10.1109/TII.2014.2300753.

[3] Vaidya, Saurabh\&Ambad, Prashant\&Bhosle, Santosh. (2018). Industry 4.0 - A Glimpse. 20. 233-238. 10.1016/j.promfg.2018.02.034.

[4] Alcacer, V., Cruz-Machado, V., 2019. Scanning industry 4.0:a literature review on technologies for manufacturing systems. Eng. Sci. Technol. Int. J. 22 (3).

[5] https://www.researchnester.com/reports/internet-ofthings-iot-market-global-demand-growth-analysisopportunity-outlook-

2023/216\#: :text=The\%20global\%20internet\%20of\%20t hings,forecast $\% 20$ period $\% 202016 \% 2$ D2023\%20globally

[6] R. Khan, S. U. Khan, R. Zaheer, and S. Khan, "Future Internet: The Internet of Things architecture, possible applications and key challenges," in Proc. 10th Int. Conf. FIT, 2012, pp. 257-260.

[7] Z. Yang et al., "Study and application on the architecture and key technologies for IoT," in Proc. ICMT, 2011, pp. 747-751.

[8] M. Wu, T. J. Lu, F. Y. Ling, J. Sun, and H. Y. Du, "Research on the architecture of Internet of Things," in Proc. 3rd ICACTE, 2010, pp. V5-484-V5-487.

[9] W. Ejaz, A. Anpalagan, M. Imran, M. Jo, M. Naeem, S. Qaisar, and W. Wang, "Internet of Things (IoT) in 5G Wireless Communications," IEEE Access, vol. 4, pp. $10310-10314,2016$.

[10] A. Al-Fuqaha, M. Guizani, M. Mohammadi, M. Aledhari, and M. Ayyash, "Internet of Things: A survey on enabling technologies, protocols, and applications," IEEE Commun. Surv. Tuts., vol. 17, no. 4, pp 2347-2376, 4th Quart., 2015.

[11] https://dzone.com/articles/iot-software-platformcomparison

[12] D. J. Cook, A. S. Crandall, B. L. Thomas, and N. C. Krishnan, “CASAS:A smart home in a box," Computer, vol. 46, no. 7, pp. 62-69, Jul. 2013. 
[13] N. Komninos, E. Philippou, and A. Pitsillides, "Survey in smart grid and smart home security: Issues, challenges, and countermeasures," IEEE Commun. Surveys Tuts., vol. 16, no. 4, pp. 1933-1954, 4th Quart. 2014.

[14] A. O. Putri, M. A. M. Ali, M. Saad, and S. S. Hidayat, "Wearable sensor and internet of things technology for better medical science: A review," International Journal of Engineering and Technology(UAE), vol. 7, no. 4, pp. $1-4,2018$.

[15] I. Ungurean, N. C. Gaitan, and V. G. Gaitan, "An IoT architecture for things from industrial environment," in Proc. 10th Int. COMM,2014,pp. 1-4.

[16] C. Wang, Z. Bi, and L. D. Xu, "IoT and cloud computing in automation of assembly modeling systems," IEEE Trans. Ind. Informat., vol. 10, no. 2, pp. 1426-1434, May 2014.

[17] https://www.sam-solutions.com/blog/internet-of-thingsiot-protocols-and-connectivity-options-an-overview/

[18] P. Sethi and Smruti R. Sarangi. Internet of things: Architectures, protocols, and applications. 2017:1-25, 01 2017.

[19] D. Culler and S. Chakrabarti, "6lowpan: incorporating IEEE 802.15. 4 into the IP architecture, IPSO Alliance," White Paper, 2009.

[20] J. Vasseur, N. Agarwal, J. Hui, Z. Shelby, P. Bertrand, and C. Chauvenet, "Rpl: the ip routing protocol designed for low power and lossy networks," Internet Protocol for Smart Objects (IPSO) Alliance 36, 2011.

[21] P. Baronti, P. Pillai, V. W. C. Chook, S. Chessa, A. Gotta, and Y. F. Hu, "Wireless sensor networks: a survey on the state of the art and the 802.15.4 and ZigBee standards," Computer Communications, vol. 30, no. 7, pp. 1655-1695, 2007

[22] W. Colitti, K. Steenhaut, N. De Caro, B. Buta, and V. Dobrota, "Evaluation of constrained application protocol for wireless sensor networks," in Proceedings of the 18th IEEE Workshop on Local and Metropolitan Area Networks (LANMAN '11), pp. 1-6, IEEE, Chapel Hill, NC, USA, October 2011.

[23] Z. Shelby, K. Hartke, and C. Bormann, "The constrained application protocol (CoAP),” Tech. Rep., IETF, 2014.

[24] D. Locke, "MQ telemetry transport (MQTT) v3. 1 protocol specification," IBM developerWorks Technical Library,2010,

http://www.ibm.com/developerworks/webservices/librar y/wsmqtt/index.html.

[25] P. Saint-Andre, "Extensible messaging and presence protocol (XMPP):Core," Internet Eng. Task Force (IETF), Fremont, CA, USA, Request for Comments: 6120, 2011.

[26] "OASIS Advanced Message Queuing Protocol (AMQP) Version 1.0,"Adv. Open Std. Inf. Soc. (OASIS), Burlington, MA, USA, 2012.

[27] Data distribution services specification, V1.2, Object Manage. Group(OMG), Needham, MA, USA, Apr. 2, 2015. [Online]. Available: http:// www.omg.org/spec/DDS/1.2/

[28] Botta, Alessio\&Donato, Walter \&Persico, Valerio
\&Pescapè, Antonio. (2015). Integration of Cloud computing and Internet of Things: A survey. Future Generation Computer Systems. 56.10 .1016 /j.future.2015.09.021.

[29] Sivakumar, S. \&Anuratha, V. \&Gunasekaran, S.. (2017). Survey on Integration of Cloud Computing and Internet of Things Using Application Perspective. International Journal of Emerging Research in Management and Technology. 6. 101-108. 10.23956/ijermt/SV6N4/101.

[30] J. Qin, Y. Liu, R. Grosvenor, A Categorical Framework of Manufacturing for Industry 4.0 and Beyond, Changeable, Agile, Reconfigurable \& Virtual Production, Procedia CIRP 52 (2016) 173 - 178

[31] https://supplychaingamechanger.com/the-industrialrevolution-from-industry-1-0-to-industry-5-0/.

[32] https://www.simio.com/blog/2018/09/05/evolutionindustrial-ages-industry-1-0-4-0/.

[33] Alcácer, V., \& Cruz-Machado, V. (Accepted/In press). Scanning the Industry 4.0: A Literature Review on Technologies for Manufacturing Systems. Engineering Science and Technology, an International Journal, 22(3), 899-919.

[34] Vaidya, Saurabh \& Ambad, Prashant \& Bhosle, Santosh. (2018). Industry 4.0 - A Glimpse. 20. 233-238. 10.1016/j.promfg.2018.02.034.

[35] Xu, Li \& He, Wu \& Li, Shancang. (2014). Internet of Things in Industries: A Survey. IEEE Transactions on Industrial Informatics. 10. 2233-2243.10.1109 /TII.2014. 2300753.

[36] Sisinni, Emiliano \& Brandao, Dennis \& Rocha, M.S. \& Rinaldi, Stefano \& Ferrari, P.. (2018). Delay Estimation of Industrial IoT Applications Based on Messaging Protocols. IEEE Transactions on Instrumentation and Measurement. 67.10.1109/TIM.2018.2813798.

[37] Modbus Application Protocol Specification V1.1b3. 2012. Available online: http://www.modbus.org/docs/ Modbus_Application_Protocol_V1_1b3.pdf (accessed on 3 February 2019).

[38] Xu, Hansong \& Yu, Wei \& Griffith, David \& Golmie, Nada. (2018). A Survey on Industrial Internet of Things: A Cyber-Physical Systems Perspective. IEEE Access. PP. 1-1. 10.1109/ACCESS.2018.2884906.

[39] Xu, Hansong \& Yu, Wei \& Griffith, David \& Golmie, Nada. (2018). A Survey on Industrial Internet of Things: A Cyber-Physical Systems Perspective. IEEE Access. PP. 1-1. 10.1109/ACCESS.2018.2884906.

[40] Falco, Gregory \& Caldera, Carlos \& Shrobe, Howard. (2018). IIoT Cybersecurity Risk Modeling for SCADA Systems. IEEE Internet of Things Journal. 1-1. 10.1109/JIOT.2018.2822842.

[41] https://www.tcs.com/blogs/the-role-of-augmentedreality-and-iot-in-the-connected-digital-enterprise

[42] N. S. C. Babu, "Keynote 1: Internet of Things(IoT) and augmented reality for e-learning," 2017 5th National Conference on E-Learning \& E-Learning Technologies (ELELTECH), Hyderabad, 2017, pp. 1-10, doi: 10.1109/ELELTECH.2017.8074987.

[43] Amanjot Singh and et. al., "Image Processing and IOT 
Based Applications", International Journal of Innovative Science and Research Technology, Volume 3, Issue 11, November - 2018.

[44] A. Kapoor, S. I. Bhat, S. Shidnal and A. Mehra, "Implementation of IoT (Internet of Things) and Image processing in smart agriculture," 2016 International Conference on Computation System and Information Technology for Sustainable Solutions (CSITSS), Bangalore, 2016, pp. 21-26, doi: 10.1109/CSITSS.2016.7779434.

[45] https://www.hitechnectar.com/blogs/examples-industrialinternet-of-things/\#Difference

[46] Ryan, Peter \& Watson, Richard. (2017). Research Challenges for the Internet of Things: What Role Can OR Play? Systems. 5. 24. 10.3390/systems5010024.

[47] L. Chettri and R. Bera, "A Comprehensive Survey on Internet of Things (IoT) Toward 5G Wireless Systems," in IEEE Internet of Things Journal, vol. 7, no. 1, pp. 1632, Jan. 2020, doi: 10.1109/JIOT.2019.2948888.
[48] B. Sharma and M. S. Obaidat, "Comparative analysis of IoT based products, technology and integration of IoT with cloud computing," in IET Networks, vol. 9, no. 2, pp. 43-47, 3 2020, doi: 10.1049/iet-net.2019.0180.

[49] M. Compare, P. Baraldi and E. Zio, "Challenges to IoTEnabled Predictive Maintenance for Industry 4.0," in IEEE Internet of Things Journal, vol. 7, no. 5, pp. 45854597, May 2020, doi: 10.1109/JIOT.2019.2957029.

[50] G. Mei, N. Xu, J. Qin, B. Wang and P. Qi, "A Survey of Internet of Things (IoT) for Geohazard Prevention: Applications, Technologies, and Challenges," in IEEE Internet of Things Journal, vol. 7, no. 5, pp. 4371-4386, May 2020, doi: 10.1109/JIOT.2019.2952593.

[51] E. Sisinni, A. Saifullah, S. Han, U. Jennehag and M. Gidlund, "Industrial Internet of Things: Challenges, Opportunities, and Directions," in IEEE Transactions on Industrial Informatics, vol. 14, no. 11, pp. 4724-4734, Nov. 2018, doi: 10.1109/TII.2018.2852491. 